\title{
ИЗУЧЕНИЕ СОСТАВА КАРДАНОЛА МЕТОДОМ ПОЛНОЙ ДВУМЕРНОЙ ГАЗОВОЙ ХРОМАТОГРАФИИ С ВРЕМЯПРОЛЕТНОЙ MACС-СПЕКTРОМЕТРИЕЙ (GCXGC-TOF MS)
}

\author{
() О.Ф. Шиилов ${ }^{1 *}$, И. Зростликова ${ }^{2}$, Д.Б. Чиркин ${ }^{3}$ \\ 'ОАО «Уралхимпласт», Северное шоссе, 21, Нижний Тагил, Свердловская \\ область, 622012 (Россия), e-mail: shishlov@ucp.ru \\ ${ }^{2}$ LECO European Technical Centre Prague, Sokolovská, 219, 190 00, Prague, 9, \\ (Czech Republic), e-mail: jitka.zrostlikova@leco.cz \\ ЗЗЗО «ЛЕКО Центр-М» Уральский филиал, ул. Бебеля, 17, Екатеринбург, \\ Свердловская область, 620034 (Россия), e-mail: chirkin@leco.ru
}

Методом GC×GC-TOF MS был изучен качественный и количественный состав такого сложного объекта как карданол. Было определено 33 соединения, относящиеся к 4 структурным группам. Основными компонентами, содержащимися в карданоле производства Southern Agro Phenols Limited (SAPL), штат Тамил Наду, Индия, являются 3-пентадеценилфенол 33,4\%, 3-пентадекадиенилфенол 24,4\%, 3-пентадекатриенилфенол 33,5\%. Технология GC ×GC обладает принципиально новой возможностью разделения, что очень полезно для разделения отдельных соединений, отличающихся числом двойных связей в боковых цепях.

Ключевые слова: карданол, хроматография, масс-спектрометрия, кардол, состав.

\section{Введение}

Жидкое топливо из растительных масел и крахмала, высокопрочные пластики из зерновых, моющие средства из сахарной свеклы, красители и дешевые витамины из растительных материалов являются примерами инновационного, постоянно набирающего обороты использования в химической промышленности возобновляемых видов сырья из растительных источников. Такие продукты находятся также в центре внимания нефте- и газодобывающих компаний $[1,2]$. Несмотря на то, что многие растения являются основой производства пищевых продуктов, использование в технических целях отходов их производства не приведет к ограничению пищевых ресурсов и расширит сырьевую базу для химической технологии и биотехнологии. Использование побочных продуктов и отходов сельского хозяйства приобретает особую важность.

Карданол, выделяемый из жидкости скорлупы орехов кешью, представляет собой перспективное сырье для производства полимеров, синтетических смол, продуктов органического синтеза $[1,3]$, в связи с чем особую важность представляет контроль состава карданола и разработка совершенных аналитических методов изучения состава данного продукта.

Кешью (Anacardium occidentale Linn) - это вечнозеленое дерево из семейства цветковых растений Anacardiaceae. Родиной данного растения является северо-восточная Бразилия, где используют его порту-

Шишлов Олег Федорович - директор по науке и развитию, кандидат технических наук, тел.: (3435) 34-65-92, факс: (3435) 34-69-85, e-mail: o.shishlov@ucp.ru Зростликова Итка (Zrostlíková Jitka) - TOF MS специалист, Ph. D., e-mail: jitka.zrostlikova @leco.cz Чиркин Дмитрий Борисович - директор, тел./факс: (343) 373-74-79, e-mail: chirkin@leco.ru гальское название Саји (плод) или Cajueiro (дерево). В настоящее время дерево широко культивируется в местах с тропическим климатом для получения орехов кешью. Кешью производится в 32 странах мира [4]. В течение многих лет лидером в производстве кешью была Индия $[7,8]$, сейчас первое место в спи-

\footnotetext{
* Автор, с которым следует вести переписку.
} 
ске производителей орехов кешью занимает Вьетнам [5, 6]. Другие страны также прилагают значительные усилия для того, чтобы развить производство кешью. В недавнее время была проведена селекция гибридов кешью в Австралии [9], Нигерии [10] и Танзании [11], Китай также входит в бизнес кешью [12]. Мировое производство жидкости скорлупы орехов кешью составляет примерно 450000 т/год.

Известно, что карданол представляет собой смесь алкилфенолов, состоящую преимущественно из 3пентадецилфенола, 3-(8(Z)-пентадеценил)фенола, 3-(8(Z),11(Z)-пентадекадиенил)фенола и 3-(8(Z),11(Z),14пентадекатриенил)-фенола [13, 14].

Широкое исследование состава жидкости скорлупы орехов кешью из разных мировых источников было проведено J.H. Tyman и L.S. Kiong [15].

Карданол представляет собой достаточно сложный объект для исследования, поэтому задача поиска современных аналитических методов является наиболее актуальной.

В последнее десятилетие современная двухмерная газовая хроматография ( $\mathrm{GC} \times \mathrm{GC})$ проявила себя как мощный метод анализа комплексных матриц. В $\mathrm{GC} \times \mathrm{GC}$ через модулятор соединены две колонки разной селективности, происходит разделение всего образца по двум независимым механизмам. B $\mathrm{GC} \times \mathrm{GC}$ увеличена пиковая емкость $[16,17]$ и улучшена возможность обнаружения анализируемого компонента $[18,19]$ по сравнению с одномерной GC. В принципе преимущества и применение $\mathrm{GC} \times \mathrm{GC}$ были описаны в недавних обзорах $[16,20-23]$. При использовании метода GC×GC-TOF MS необходим достаточно быстродействующий масс-спектрометр, позволяющий описать очень узкие пики (обычно 0,1-0,3 c), получаемые при данной методике разделения. Поэтому в большинстве исследований выбирают высокоскоростную методику TOF MS [18].

Цель данного исследования - изучение состава карданола, производимого Southern Agro Phenols Limited (SAPL), штат Тамил Наду, Индия, методом двумерной газовой хроматографии с времяпролетной масс-спектрометрией (GC×GC-TOF MS).

\section{Экспериментальная часть}

Для проведения исследования использовалась полная двумерная газовая хроматография с времяпролетным масс-спектрометром (GC $\times$ GC-TOF MS), система Pegasus 4D, производство компании LECO Corporation, St. Joseph, MI, USA. Эта система состоит из газового хроматографа Agilent 7890A с встроенной второй печью, двухстадийным струйным криомодулятором и времяпролетным масс-анализатором Pegasus HT (LECO). Обработка данных проводилась с помощью программы Chroma TOF (LECO).

Условия проведения анализа следующие:

Масс-спектрометр: температура источника ионов (ЕI ионизация) $220^{\circ} \mathrm{C}$, частота 100 Гц, массовый диапазон 35-500, напряжение детектора 1450 В.

Двумерная газовая хроматография: инжектор: температура $340{ }^{\circ} \mathrm{C}$, газ-носитель Не, скорость потока 1 мл/мин, колонка 1Rxi-5 (30 m × 0,25 mm × 0,25 $\mu \mathrm{m})$, колонка 2 BPX-50 $(1,5 \mathrm{~m} \times 0,1 \mathrm{~mm} \times 0,1 \mu \mathrm{m})$, программа температуры главной печи: $100^{\circ} \mathrm{C}\left(1\right.$ мин), $8{ }^{\circ} \mathrm{C} /$ мин до $300{ }^{\circ} \mathrm{C}(8$ мин), программа температуры второй печи: на $10^{\circ} \mathrm{C}$ выше температуры главной печи на протяжении всего времени анализа, программа модулятора: на $30^{\circ} \mathrm{C}$ выше температуры главной печи на протяжении всего времени анализа, период модуляции $3 \mathrm{c}$.

Образец карданола растворяли в гексане в соотношении $1: 10$.

\section{Обсуждение результатов}

Данные 2D анализа были обработаны с помощью программного обеспечения Chroma TOF, которое автоматически определяет пики во всех полученных отдельных массах выше определенного уровня отношения «сигнал (С)/шум (Ш)» (в данном случае использовался показатель С/Ш >200). После этого деконволюционный алгоритм математически отделяет массовый спектр от хроматографически извлеченных из адсорбента соединений. Модулированные пики автоматически комбинируются, а их массовые спектры сравниваются с библиотекой NIST MS для автоматической идентификации. В результате создается таблица пиков с наименованиями определенных соединений.

Четыре основные группы соединений, определенные в образце карданола, приведены в таблице 1. 
А. Фенолы (карданол), содержащие алифатический углеводородный заместитель С11-С17. Заместитель имеет от 0 до 3 двойных связей.

B. Дигидроксибензолы (кардол), содержащие алифатический углеводородный заместитель С11С15. Заместитель имеет от 0 до 3 двойных связей.

С. Фенолы (метилкарданол), содержащие алифатический углеводородный заместитель С15. Заместитель имеет от 0 до 3 двойных связей.

D. Дигидроксибензолы (метилкардол), содержащие алифатический углеводородный заместитель C13-С15. Заместитель имеет от 0 до 3 двойных связей.

Формулы данных соединений изображены на рисунке 1.<smiles>[R]c1cccc(O)c1</smiles>

A<smiles>[R]c1cc(O)cc(O)c1</smiles>

B<smiles>[R]c1cc(C)cc(O)c1</smiles>

C<smiles>[R]c1cc(O)c(C)c(O)c1</smiles>

D

Рис. 1. Формулы соединений, определенных в образце карданола. Маркировка буквами A, B, C, D соответствует отдельным группам соединений, приведенным в таблице 1

Таблица 1. Соединения, определенные в образце карданола

\begin{tabular}{|c|c|c|c|c|c|}
\hline $\begin{array}{c}\text { Наименование } \\
\text { группы }\end{array}$ & $\begin{array}{c}\text { № } \\
\text { пика }\end{array}$ & $\begin{array}{l}\text { Число атомов угле- } \\
\text { рода в заместителе }\end{array}$ & $\begin{array}{l}\text { Количество двойных } \\
\text { связей в заместителе }\end{array}$ & $\begin{array}{c}\text { Основной фраг- } \\
\text { ментный ион }\end{array}$ & $\begin{array}{c}\text { Молекулярный } \\
\text { ион }\end{array}$ \\
\hline \multirow{17}{*}{$\begin{array}{c}\text { Фенолы } \\
\text { (карданол) }\end{array}$} & A1 & 11 & 0 & \multirow{9}{*}{108} & 248 \\
\hline & $\mathrm{A} 2$ & 13 & 0 & & 276 \\
\hline & A3 & 13 & 1 & & 274 \\
\hline & A4 & 14 & 0 & & 290 \\
\hline & A5 & 14 & 1 & & 288 \\
\hline & A6 & 14 & 2 & & 286 \\
\hline & A7 & 15 & 0 & & 304 \\
\hline & A8 & 15 & 1 & & 302 \\
\hline & A9 & 15 & 2 & & 300 \\
\hline & A10 & 15 & 3 & 79,107 & 298 \\
\hline & A11 & 16 & 0 & \multirow{6}{*}{108} & 318 \\
\hline & $\mathrm{A} 12$ & 16 & 1 & & 316 \\
\hline & A13 & 16 & 2 & & 314 \\
\hline & A15 & 17 & 0 & & 332 \\
\hline & A16 & 17 & 1 & & 330 \\
\hline & A17 & 17 & 2 & & 328 \\
\hline & A18 & 17 & 3 & 79,107 & 326 \\
\hline \multirow{6}{*}{$\begin{array}{c}\text { Дигидроксибензолы } \\
\text { (кардол) }\end{array}$} & B1 & 11 & 0 & \multirow{6}{*}{124} & 264 \\
\hline & B2 & 13 & 0 & & 292 \\
\hline & B3 & 15 & 0 & & 320 \\
\hline & B4 & 15 & 1 & & 318 \\
\hline & B5 & 15 & 2 & & 316 \\
\hline & B6 & 15 & 3 & & 314 \\
\hline \multirow{4}{*}{$\begin{array}{c}\text { Фенолы } \\
\text { (метилкарданол) }\end{array}$} & $\mathrm{C} 1$ & 15 & 0 & \multirow{4}{*}{122} & 318 \\
\hline & $\mathrm{C} 2$ & 15 & 1 & & 316 \\
\hline & $\mathrm{C} 3$ & 15 & 2 & & 314 \\
\hline & $\mathrm{C} 4$ & 15 & 3 & & 312 \\
\hline \multirow{5}{*}{$\begin{array}{c}\text { Дигидроксибензолы } \\
\text { (метилкардол) }\end{array}$} & D1 & 13 & 0 & \multirow{5}{*}{138} & 306 \\
\hline & D2 & 15 & 0 & & 334 \\
\hline & D3 & 15 & 1 & & 332 \\
\hline & D4 & 15 & 2 & & 330 \\
\hline & D5 & 15 & 3 & & 328 \\
\hline
\end{tabular}


Масс-спектры основных соединений, входящих в состав карданола, показаны на рисунке 2. Пик молекулярного иона, соответствующий молекулярной массе соединения, для моно-, ди- и три- ненасыщенных производных м-пентадецилфенола равен 302, 300 и 298 соответственно.

Исследуемый образец содержал в своей структуре родственные соединения, имеющие тенденцию к совместному элюированию. Один из примеров представлен на рисунке 3. Отдельные компоненты кардола (B4, B5, В6), отличающиеся друг от друга только количеством двойных связей в заместителе, при проведении анализа при помощи одномерной газовой хроматографии элюируются совместно и количественное определение их не может быть проведено.
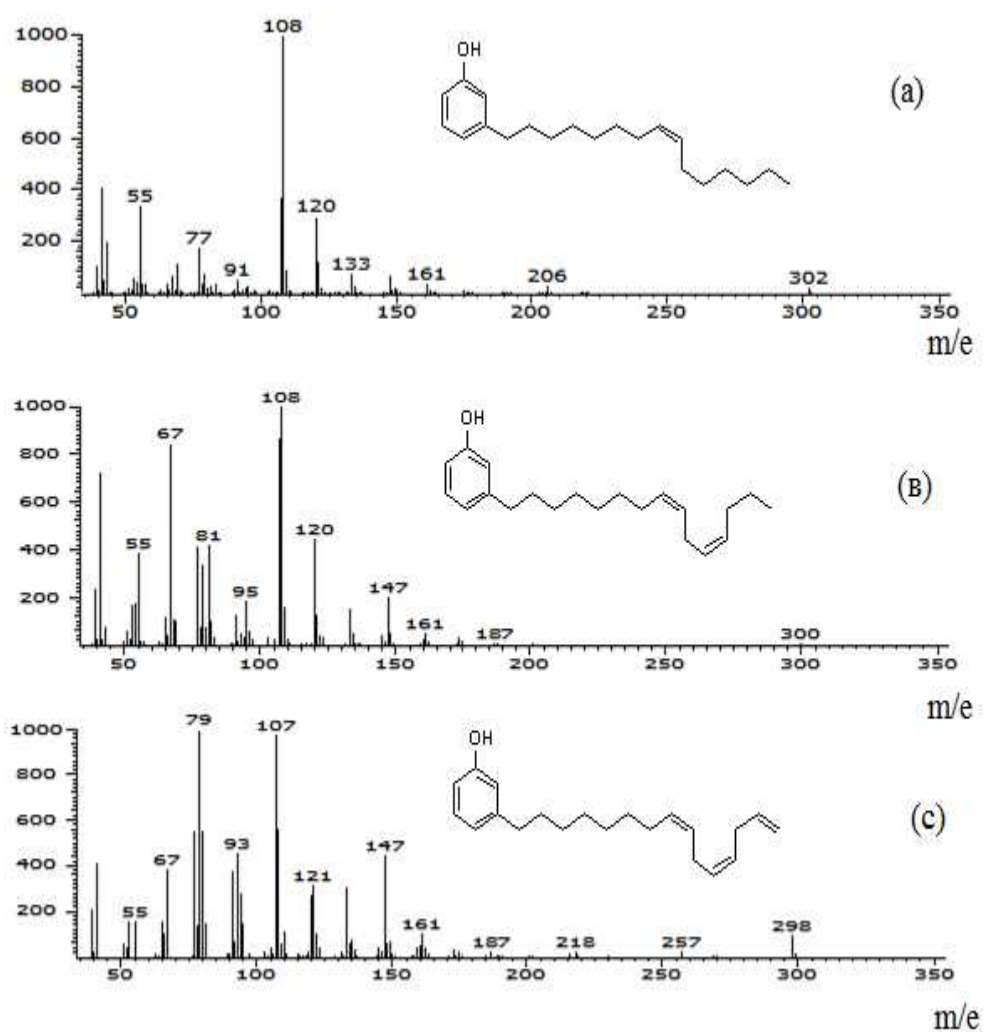

Рис. 2. Масс-спектры

3-пентадеценилфенола (а), 3-пентадекадиенилфенола (в), 3-пентадекатриенилфенола (c)

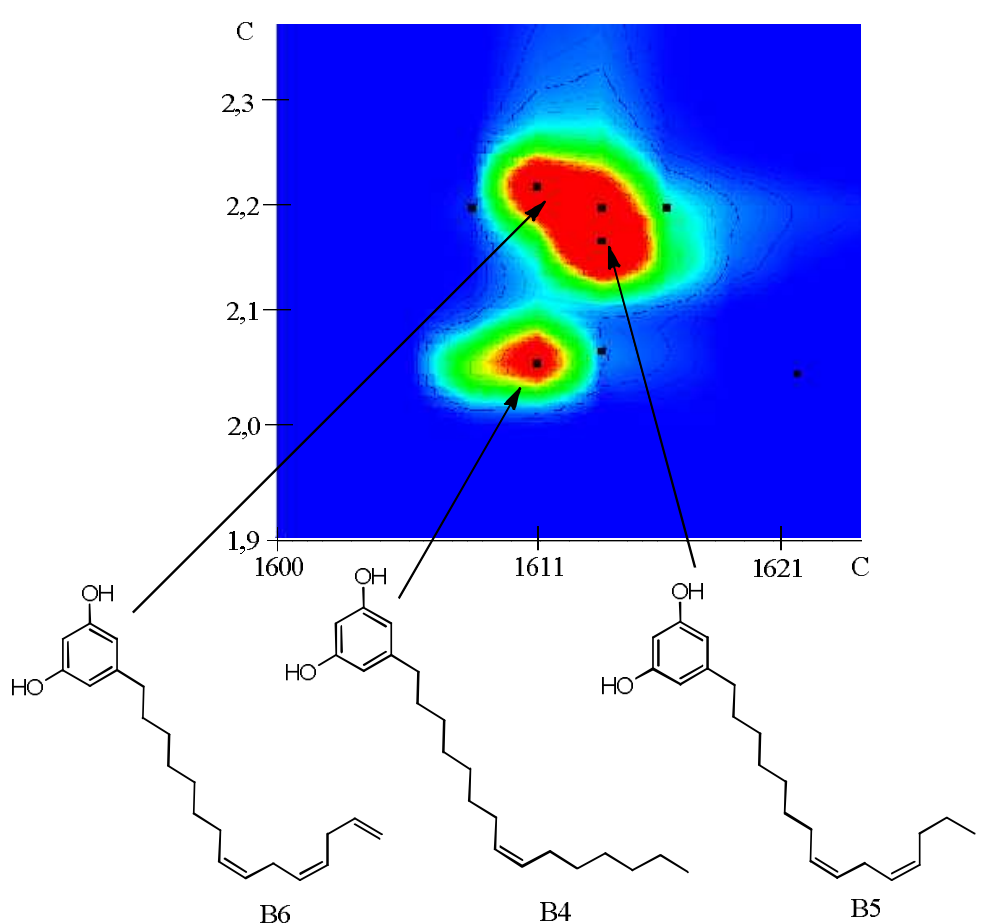

Рис. 3. GC $\times \mathrm{GC}$ разделение компонентов кардола (B4 - 5-пентадеценил-1,3-дигидроксибензол, В5 - 5-пентадекадиенил-1,3-дигидроксибензол, В6 - 5-пентадекатриенил-1,3-дигидроксибензол) 
При GC× GC в рамках одного анализа используются две колонки с разной селективностью, в результате чего достигается гораздо более эффективное хроматографическое разделение. В этом отдельном случае три соединения, несмотря на то, что они были лишь частично разделены при первом измерении (неполярная колонка), разделились во втором измерении (полярная колонка) и показаны на рисунке 3.

Подобная ситуация возникла также и с отдельными соединениями замещенных фенолов (карданол), отличающихся количеством двойных связей в заместителе. При одномерной хроматографии четыре соединения (A7-А10) разделяются только частично, вследствие чего их точное количественное определение неспецифическим детектором практически невозможно.

При использовании GC×GC-TOF MS было получено два дополнительных уровня информации:

1) большее хроматографическое разделение при помощи $\mathrm{GC} \times \mathrm{GC}$;

2) информация о масс-спектрах, позволяющая использовать для количественного определения удельный вес.

В результате исследования было проведено количественное определение соединений, имеющихся в наибольшем количестве. Результаты представлены в таблице 2.

Таблица 2. Количественный состав карданола

\begin{tabular}{|c|c|c|c|c|c|c|}
\hline $\begin{array}{l}\text { № } \\
\text { пика }\end{array}$ & $\begin{array}{c}\text { Время удер- } \\
\text { живания, } \\
\text { колонка } 1, \mathrm{c}\end{array}$ & $\begin{array}{l}\text { Время удер- } \\
\text { живания, } \\
\text { колонка 2, с }\end{array}$ & Наименование & $\begin{array}{c}\text { Количество атомов } \\
\text { углерода в алкиль- } \\
\text { ном заместителе }\end{array}$ & $\begin{array}{c}\text { Количест- } \\
\text { во двой- } \\
\text { ных связей }\end{array}$ & $\begin{array}{c}\text { Содер- } \\
\text { жание, } \\
\%\end{array}$ \\
\hline A1 & 1200 & 1,420 & 3-ундецилфенол & 11 & 0 & 0,1 \\
\hline A2 & 1332 & 1,450 & 3-тридецилфенол & 13 & 0 & 0,7 \\
\hline A5 & 1389 & 1,510 & 3-тетрадеценилфенол & 14 & 1 & 0,1 \\
\hline $\mathrm{C} 2$ & 1410 & 1,420 & $\begin{array}{c}\text { 3-пентадеценил-5- } \\
\text { метилфенол }\end{array}$ & 15 & 1 & 0,1 \\
\hline $\mathrm{C} 4$ & 1410 & 1,494 & $\begin{array}{c}\text { 3-пентадекатриенил-5- } \\
\text { метилфенол }\end{array}$ & 15 & 3 & 0,1 \\
\hline A8 & 1446 & 1,580 & 3-пентадеценилфенол & 15 & 1 & 33,4 \\
\hline A10 & 1446 & 1,660 & 3-пентадекатриенил-фенол & 15 & 3 & 33,5 \\
\hline A9 & 1449 & 1,619 & 3-пентадекадиенил-фенол & 15 & 2 & 24,4 \\
\hline A7 & 1455 & 1,490 & 3-пентадецилфенол & 15 & 0 & 2,0 \\
\hline A17 & 1554 & 1,650 & 3-гептадекадиенил-фенол & 17 & 2 & 0,3 \\
\hline A16 & 1560 & 1,610 & 3-гептадеценилфенол & 17 & 1 & 1,0 \\
\hline A18 & 1560 & 1,710 & 3-гептадекатриенил-фенол & 17 & 3 & 1,1 \\
\hline B4 & 1611 & 2,050 & $\begin{array}{l}\text { 5-пентадеценил-1,3- } \\
\text { дигидроксибензол }\end{array}$ & 15 & 1 & 0,5 \\
\hline B6 & 1611 & 2,200 & $\begin{array}{c}\text { 5-пентадекатриенил-1,3- } \\
\text { дигидроксибензол }\end{array}$ & 15 & 3 & 1,3 \\
\hline B5 & 1614 & 2,160 & $\begin{array}{c}\text { 5-пентадекадиенил-1,3- } \\
\text { дигидроксибензол }\end{array}$ & 15 & 2 & 1,3 \\
\hline
\end{tabular}

\section{Bblвodbl}

1. Методом GC×GC-TOF MS был определен качественный и количественный состав такого сложного объекта, как карданол. Автоматический поиск пиков и идентификационные алгоритмы программного обеспечения Chroma TOF представляют собой отличный инструмент для скоростной идентификации. Было определено 33 соединения, относящиеся к 4 структурным группам, а также получены масс-спектры достаточно хорошего качества.

2. Основными компонентами, содержащимися в карданоле производства Southern Agro Phenols Limited (SAPL), штат Тамил Наду, Индия, являются 3-пентадеценилфенол 33,4\%, 3-пентадекадиенилфенол 24,4\%, 3-пентадекатриенилфенол 33,5\%. Показано, что количество атомов углерода в м-алкильном заместителе представляет собой нечетное число $(11,13,15,17)$. Суммарное количество алкилзамещенных производных резорцина (кардол) составляет 3,1\% и имеет следующее распределение: 5-пентадеценил-1,3дигидроксибензол $\quad 0,5 \%, \quad 5$-пентадекадиенил-1,3-дигидроксибензол $1,3 \%$ и 5-пентадекатриенил-1,3дигидроксибензол $1,3 \%$. Кардол обладает повышенной реакционной способностью, в связи с чем его количественное определение представляет важную практическую задачу. 
3. Технология $\mathrm{GC} \times \mathrm{GC}$ обладает принципиально новой возможностью разделения, что очень полезно для разделения отдельных соединений, отличающихся числом двойных связей в боковых цепях. При использовании традиционной технологии отдельные соединения элюируются совместно, в результате чего точное количественное определение становится невозможным. GC $\times \mathrm{GC}$ способна разделять критичные пары, благодаря чему возможно точное количественное определение.

\section{Список литературы}

1. Talbiersky J., Polaczek J., Ramamoorty R., Shishlov O. Phenols from Cashew Nut Shell Oil as a Feedstock for Making Resins and Chemicals // OIL GAS Europeen Magazine. 2009. N1. Pp. 33-39.

2. Lubi M.C., Thachil E.T. Cashew nut shell liquid (CNSL) - a versatile monomer for polymer synthesis // Designed Monomers and Polymers. 2000. Vol. 3, N2. Pp. 123-153.

3. Шишлов О.Ф., Глухих В.В. Синтез, свойства и применение продуктов поликонденсации карданола с формальдегидом (обзор) // Химия растительного сырья. 2011. №1. С. 5-16.

4. Cashew. URL: http://en.wikipedia.org/wiki/Cashew

5. Vietnam posing threat to Indian cashew exports, Asia Pulse News. 2004, 24 September.

6. Nair G.K. Cashew kernel exports decline, Business Line. 2004. May 13.

7. Nair G.K. Vietnam will overtake India in cashew market soon. URL: http://sify.com/finance/fullstory.php?id=14567037 (2007, Nov. 27)

8. Cashew nut shell liquid (CNSL) // Small Industries Service Instituite, Guindy. 2003.

9. Blaikie S., O'Farrell P., Müller W., Wei X., Scott N., Sykes S., Chacko E. Assesment and selection of new cashew hybrids. A report for the Rural Industries Research and Development Corporation. January 2002.

10. Aliyu O.M. Clonal propagation in cashew (Anacardiumoccidentale): effect of rooting media on the rootability and sprouting of air-layers // Tropical Sci. 2007. Vol. 47. Pp. 65-72.

11. Massawe P.A.L., Mfume S., Mbunda Z., Performance of cashew hybrids developed from partial dialed crossing between selected clones in Tanzania // Research \& Training Newsletter. 2005. Vol. 6, N1-4. Pp. 5-8.

12. Shantou Chaoyang Chengnan Cashew Nut Shell Oil Factory. URL: http://www.chengnan.com.cn/doce/gsjj.htm.

13. Symes W.F., Dawson C.R., Separation and structural determination of the olefinic components, of poison ivy urushiol, cardanol and cardol // Nature. 1953. Vol. 171. P. 841.

14. Paul V.J., Yeddanapalli L.M., Olefinic nature of anacardic acid from Indian cashew nut shell liquid // Nature. 1954. Vol. 174. P. 604.

15. Tyman J.H., Kiong L.S., Long chain phenols: Part XI. Composition of natural cashew nutshell liquid (Anacardiumoccidentale) from various sources // Lipids. 1978. Vol. 13. N8. Pp. 525-532.

16. Adahchour M., Beens J., Vreuls R.J.J., Brinkmann U.A.Th. Recent developments in comprehensive two-dimensional gas chromatography (GC×GC): I. Introduction and instrumental set-up // Tr. Anal. Chem. 2006. Vol. 25. Pp. 438-454.

17. Bertsch W. Two-Dimensional Gas Chromatography. Concepts, Instrumentation, and Applications - Part 2: Comprehensive Two-Dimensional Gas Chromatography // J. High Resolut. Chromatogr. 2000. Vol. 23. Pp. 167-181.

18. Dallüge J., Vreuls R.J.J., Beens J., Brinkmann U.A.Th. Optimization and characterization of comprehensive twodimensional gas chromatography with time-of-flight mass spectrometric detection (GC×GC-TOF MS) // J. Sep. Sci. 2002. Vol. 25. Pp. 201-214.

19. Zrostlíková J., Hajšlová J., Čajka T. Evaluation of two-dimensional gas chromatography-time-of-flight mass spectrometry for the determination of multiple pesticide residues in fruit // J. Chromatogr. A. 2003. Vol. 1019. Pp. 173-186.

20. Adahchour M., Beens J., Brinkmann U.A.Th. Recent developments in the application of comprehensive twodimensional gas chromatography // J. Chromatogr. A. 2008. Vol. 1186. Pp. 67-108.

21. Adahchour M., Beens J., Vreuls R.J.J., Brinkmann U.A.Th. Recent developments in comprehensive two-dimensional gas chromatography $(\mathrm{GC} \times \mathrm{GC})$ : II. Modulation and detection // Tr. Anal. Chem. 2006. Vol. 25. Pp. 540-553.

22. Adahchour M., Beens J., Vreuls R.J.J., Brinkmann U.A.Th. Recent developments in comprehensive two-dimensional gas chromatography $(\mathrm{GC} \times \mathrm{GC})$ : III. Applications for petrochemicals and organohalogens // Tr. Anal. Chem. 2006. Vol. 25. Pp. 726-741.

23. Adahchour M., Beens J., Vreuls R.J.J., Brinkmann U.A.Th. Recent developments in comprehensive two-dimensional gas chromatography $(\mathrm{GC} \times \mathrm{GC})$ : II. Modulation and detection // Tr. Anal. Chem. 2006. Vol. 25. Pp. 821-840. 
Shishlov O.F. ${ }^{l^{*}}$, Zrostlikova I. ${ }^{2}$, Chirkin D.B. ${ }^{3}$ Investigating of cardanol composition with two dimensional full gas chromatography with time of flight mass-spectrometry technique (GCxGC-TOF MS)

${ }^{1}$ Uralchimplast Ltd., Severnoe shosse, 21, Nizhny Tagil, Sverdlovsk region, 622012 (Russia), e-mail: shishlov@ucp.ru

${ }^{2}$ LECO European Technical Centre Prague, Sokolovská, 219, 190 00, Prague, 9, (Czech Republic), e-mail: jitka.zrostlikova@leco.cz.

${ }^{3}$ LECO Ural branch, ul. Bebelia,17, Yekaterinburg, Sverdlovsk Region, 620034 (Russia), e-mail: chirkin@leco.ru

With the use of GCxGC-TOF MS technique qualitative and quantitative composition of a complicated material - cardanol was investigated. The presence of 33 substances belonging to 4 structural groups was established. Main constituents of cardanol produced by Southern Agro Phenols Limited (SAPL), Tamil Nadu state, India were found to be: 3-pentadecylphenol 33,4\%, 3-pentadecadienylphenol - 24,4\%, 3-pentadecatrienylphenol - 33,5\%. GC $\times \mathrm{GC}$ method possess a brand new possibility on dividing components, which is very useful for isolating of individual substances varying in number of double bonds in side chains.

Keywords: cardanol, chromatography, mass-spectrometry, cardol, composition

\section{References}

1. Talbiersky J., Polaczek J., Ramamoorty R., Shishlov O. OIL GAS Europeen Magazine, 2009, no. 1, pp. 33-39.

2. Lubi M.C., Thachil E.T. Designed Monomers and Polymers, 2000, vol. 3, no. 2, pp. 123-153.

3. Shishlov O.F., Glukhikh V.V. Khimiia rastitel'nogo syr'ia, 2011, no. 1, pp. 5-16. (in Russ.).

4. Cashew. URL: http://en.wikipedia.org/wiki/Cashew

5. Vietnam posing threat to Indian cashew exports, Asia Pulse News. 2004, 24 September.

6. Nair G.K. Cashew kernel exports decline, Business Line. 2004. May 13.

7. Nair G.K. Vietnam will overtake India in cashew market soon. URL: http://sify.com/finance/fullstory.php?id=14567037 (2007, Nov. 27)

8. Cashew nut shell liquid (CNSL) // Small Industries Service Instituite, Guindy. 2003.

9. Blaikie S., O'Farrell P., Müller W., Wei X., Scott N., Sykes S., Chacko E. Assesment and selection of new cashew hybrids. A report for the Rural Industries Research and Development Corporation. January 2002.

10. Aliyu O.M. Tropical Sci., 2007, vol. 47, pp. 65-72.

11. Massawe P.A.L., Mfume S., Mbunda Z. Research \& Training Newsletter, 2005, vol. 6, no. 1-4, pp. 5-8.

12. Shantou Chaoyang Chengnan Cashew Nut Shell Oil Factory. URL: http://www.chengnan.com.cn/doce/gsjj.htm.

13. Symes W.F., Dawson C.R. Nature, 1953, vol. 171, p. 841.

14. Paul V.J., Yeddanapalli L.M. Nature, 1954, vol. 174, p. 604.

15. Tyman J.H., Kiong L.S. Lipids, 1978, vol. 13, no. 8, pp. 525-532.

16. Adahchour M., Beens J., Vreuls R.J.J., Brinkmann U.A.Th. Tr. Anal. Chem., 2006, vol. 25, pp. $438-454$.

17. Bertsch W. J. High Resolut. Chromatogr., 2000, vol. 23, pp. 167-181.

18. Dallüge J., Vreuls R.J.J., Beens J., Brinkmann U.A.Th. J. Sep. Sci., 2002, vol. 25, pp. 201-214.

19. Zrostlíková J., Hajšlová J., Čajka T. J. Chromatogr. A,2003, vol. 1019, pp. 173-186.

20. Adahchour M., Beens J., Brinkmann U.A.Th. J. Chromatogr. A, 2008, vol. 1186, pp. 67-108.

21. Adahchour M., Beens J., Vreuls R.J.J., Brinkmann U.A.Th. Tr. Anal. Chem., 2006, vol. 25, pp. 540-553.

22. Adahchour M., Beens J., Vreuls R.J.J., Brinkmann U.A.Th. Tr. Anal. Chem., 2006, vol. 25, pp. 726-741.

23. Adahchour M., Beens J., Vreuls R.J.J., Brinkmann U.A.Th. Tr. Anal. Chem., 2006, vol. 25, pp. 821-840.

\footnotetext{
* Corresponding author.
} 
